\title{
The Influence of Hormonal Factors on the Risk of Developing Cervical Cancer and Pre- Cancer: Results from the EPIC Cohort
}

\author{
Esther Roura $^{1,2 *}$, Noémie Travier ${ }^{3}$, Tim Waterboer ${ }^{4}$, Silvia de Sanjosé ${ }^{1,2}$, F. Xavier Bosch ${ }^{1}$, \\ Michael Pawlita ${ }^{4}$, Valeria Pala ${ }^{5}$, Elisabete Weiderpass $\mathbf{S}^{6,7,8,9}$, Núria Margall $^{10}$, \\ Joakim Dillner ${ }^{6,11}$, Inger T. Gram ${ }^{7}$, Anne Tjønneland ${ }^{12}$, Christian Munk ${ }^{13}$, Domenico Palli ${ }^{14}$, \\ Kay-Tee Khaw $^{15}$, Kim Overvad ${ }^{16}$, Françoise Clavel-Chapelon ${ }^{17,18,19}$, Sylvie Mesrine ${ }^{17,18,19}$, \\ Agnès Fournier ${ }^{17,18,19}$, Renée T. Fortner ${ }^{20}$, Jennifer Ose ${ }^{20}$, Annika Steffen ${ }^{21}$, \\ Antonia Trichopoulou ${ }^{22}$, Pagona Lagiou ${ }^{23,24}$, Philippos Orfanos ${ }^{22,23}$, Giovanna Masala ${ }^{14}$, \\ Rosario Tumino ${ }^{25}$, Carlotta Sacerdote ${ }^{26,27}$, Silvia Polidoro ${ }^{28}$, Amalia Mattiello ${ }^{29}$, \\ Eiliv Lund $^{7}$, Petra H. Peeters ${ }^{30,31}$, H. B(as). Bueno-de-Mesquita ${ }^{32,33,34,35}$, J. \\ Ramón Quirós ${ }^{36}$, María-José Sánchez ${ }^{2,37}$, Carmen Navarro ${ }^{2,38,39}$, Aurelio Barricarte ${ }^{2,40,41}$, \\ Nerea Larrañaga ${ }^{2,42}$, Johanna Ekström ${ }^{43}$, David Lindquist ${ }^{44}$, Annika Idahl ${ }^{45}$, \\ Ruth C. Travis ${ }^{46}$, Melissa A. Merritt ${ }^{34}$, Marc J. Gunter ${ }^{34}$, Sabina Rinaldi ${ }^{47}$, \\ Massimo Tommasino ${ }^{47}$, Silvia Franceschi ${ }^{47}$, Elio Riboli ${ }^{34}$, Xavier Castellsagué ${ }^{1,2}$
}

1 Unit of Infections and Cancer, Cancer Epidemiology Research Program, Catalan Institute of Oncology (ICO)-IDIBELL, L'Hospitalet de Llobregat, Barcelona, Catalonia, Spain, 2 CIBER de Epidemiología y Salud Pública (CIBERESP), Madrid, Spain, 3 Unit of Nutrition, Environment and Cancer, Cancer Epidemiology Research Program, Catalan Institute of Oncology (ICO)-IDIBELL, L'Hospitalet de Llobregat, Barcelona, Catalonia, Spain, 4 Division of Molecular Diagnostics of Oncogenic Infections (F020), Research Program Infection, Inflammation and Cancer, German Cancer Research Center (DKFZ), Heidelberg, Germany, 5 Epidemiology and Prevention Unit, Fondazione IRCCS Istituto Nazionale dei Tumori, Milan, Italy, 6 Department of Medical Epidemiology and Biostatistics, Karolinska Institutet, Stockholm, Sweden, 7 Department of Community Medicine, Faculty of Health Sciences, UiT The Arctic University of Norway, Troms $\varnothing$, Norway, 8 Department of Research, Cancer Registry of Norway, Oslo, Norway, 9 Genetic Epidemiology Group, Folkhälsan Research Center, Helsinki, Finland, 10 Microbiology Department, Universitat Autònoma de Barcelona, Hospital de la Santa Creu i Sant Pau, Barcelona, Catalonia, Spain, 11 Department of Laboratory Medicine, Karolinska Institutet, Stockholm, Sweden, 12 Diet, Genes and Environment, Danish Cancer Society Research Center, Copenhagen, Denmark, 13 Virus, Lifestyle and Genes, Danish Cancer Society Research Center, Copenhagen, Denmark, 14 Molecular and Nutritional Epidemiology Unit, Cancer Research and Prevention Institute-ISPO, Florence, Italy, 15 School of Clinical Medicine, University of Cambridge, Cambridge, United Kingdom, 16 Section for Epidemiology, Department of Public Health, Aarhus University, Aarhus, Denmark, 17 Inserm, Centre for research in Epidemiology and Population Health (CESP), U318, Nutrition, Hormones and Women's Health team, F-94705, Villejuif, France, 18 Université Paris Sud, UMRS 318, F-94705, Villejuif, France, 19 Institut Gustave Roussy, F-94705, Villejuif, France, 20 Division of Cancer Epidemiology, German Cancer Research Center (DKFZ), Heidelberg, Germany, 21 Department of Epidemiology, German Institute of Human Nutrition Potsdam-Rehbruecke, Nuthetal, Germany, 22 Hellenic Health Foundation, Athens, Greece, 23 Department of Hygiene, Epidemiology and Medical Statistics, University of Athens Medical School, Athens, Greece, 24 Department of Epidemiology, Harvard School of Public Health, Boston, United States of America, 25 Cancer Registry and Histopathology Unit, "Civic-M.P. Arezzo" Hospital, Ragusa, Italy, 26 Unit of Cancer Epidemiology, University of Turin, Turin, Italy, 27 Centre for Cancer Epidemiology and Prevention (CPO Piemonte), Turin, Italy, 28 Human Genetics Foundation (HuGeF), Turin, Italy, 29 Dipartimento Di Medicina Clinica E Chirurgia, Federico II University, Naples, Italy, 30 MRC-PHE Centre for Environment and Health, Department of Epidemiology and Biostatistics, School of Public Health, Imperial College, London, United Kingdom, 31 Department of Epidemiology, Julius Center for Health Sciences and Primary Care, University Medical Center Utrecht, Utrecht, The Netherlands, 32 Department for Determinants of Chronic Diseases (DCD), National Institute for Public Health and the Environment (RIVM), Bilthoven, The Netherlands, 33 Department of Gastroenterology and Hepatology, University Medical Centre, Utrecht, The Netherlands, 34 Department of Epidemiology and Biostatistics, The School of Public Health, Imperial College London, London, United Kingdom, 35 Department of Social \& Preventive Medicine, Faculty of Medicine, University of Malaya, Kuala Lumpur, Malaysia, 36 Public Health Directorate, Asturias, Spain, 37 Escuela Andaluza de 
2005SGR00695, 2009SGR939 and 2009SGR126, 2014SGR1077, 2014SGR2016). The coordination of EPIC is financially supported by the European Commission (DG-SANCO) and the International Agency for Research on Cancer. The national cohorts are supported by the Health Research Fund (FIS) of the Spanish Ministry of Health (Exp P10710130), Regional Governments of Andalucía, Asturias, Basque Country, Murcia (no. 6236), Navarra and the Catalan Institute of Oncology, La Caixa (BM 06-130), RTICC-RD06/10091 (Spain); Danish Cancer Society (Denmark); Ligue contre le Cancer, Institut Gustave Roussy, Mutuelle Générale de l'Education Nationale, Institut National de la Santé et de la Recherche Médicale (INSERM) (France); Deutsche Krebshilfe, Deutsches Krebsforschungszentrum and Federal Ministry of Education and Research (Germany); the Hellenic Health Foundation (Greece); Italian Association for Research on Cancer (AIRC) and National Research Council (Italy); Dutch Ministry of Public Health, Welfare and Sports (VWS), Netherlands Cancer Registry (NKR), LK Research Funds, Dutch Prevention Funds, Dutch ZON (Zorg Onderzoek Nederland), World Cancer Research Fund (WCRF) and Statistics Netherlands (The Netherlands); Swedish Cancer Society, Swedish Scientific Council and Regional Government of Skåne and Västerbotten (Sweden); Cancer Research UK, Medical Research Council (United Kingdom); Norwegian Research Council, Norwegian Cancer Society, University of Tromso (Norway). The funders had no role in study design, data collection and analysis, decision to publish, or preparation of the manuscript.

Competing Interests: The authors have declared that no competing interests exist.

Abbreviations: $\mathrm{CC}$, Cervical Cancer; $\mathrm{Cl}$, Confidence Interval; CIN3, Cervical Intraepithelial Neoplasia grade 3; CIS, Carcinoma In Situ; CT, Chlamydia trachomatis; EPIC, European Prospective Investigation into Cancer and Nutrition; FTP, Full-term pregnancy; HHV-2, Human herpesvirus 2; HPV, Human Papillomavirus; HR, Hazard Ratio; HT, Hormone Therapy; IARC, International Agency for Research on Cancer; ICC, Invasive Cervical Cancer; IUD, Intrauterine Device; OR, Odds Ratio; OC, Oral Contraceptives; SCC, Squamous Cell Carcinoma; STIs, Sexually Transmitted Infections.
Salud Pública, Instituto de Investigación Biosanitaria ibs.GRANADA, Hospitales Universitarios de Granada/ Universidad de Granada, Granada, Spain, 38 Department of Epidemiology, Murcia Regional Health Council, IMIB-Arrixaca, Murcia, Spain, 39 Department of Health and Social Sciences, Universidad de Murcia, Murcia, Spain, 40 Navarra Public Health Institute, Pamplona, Spain, 41 Navarra Institute for Health Research (IdiSNA), Pamplona, Spain, 42 Public Health Division of Gipuzkoa, BIODonostia Research Institute, Basque Health Department, Bilbao, Spain, 43 BBMRI.se Service Center for Southern Sweden, Lund University, Medicon Village, Lund, Sweden, 44 Department of Radiation Sciences, Umeå University, Umeå, Sweden, 45 Department of Clinical Sciences, Obstetrics and Gynecology, Umeå University, Umeå, Sweden, 46 Cancer Epidemiology Unit, Nuffield Department of Population Health, University of Oxford, Oxford, United Kingdom, 47 International Agency for Research on Cancer, Lyon, France

* eroura@iconcologia.net

\section{Abstract}

\section{Background}

In addition to HPV, high parity and hormonal contraceptives have been associated with cervical cancer (CC). However, most of the evidence comes from retrospective case-control studies. The aim of this study is to prospectively evaluate associations between hormonal factors and risk of developing cervical intraepithelial neoplasia grade 3 (CIN3)/carcinoma in situ (CIS) and invasive cervical cancer (ICC).

\section{Methods and Findings}

We followed a cohort of 308,036 women recruited in the European Prospective Investigation into Cancer and Nutrition (EPIC) Study. At enrollment, participants completed a questionnaire and provided serum. After a 9-year median follow-up, 261 ICC and $804 \mathrm{CIN} 3 / \mathrm{CIS}$ cases were reported. In a nested case-control study, the sera from 609 cases and 1,218 matched controls were tested for $L 1$ antibodies against HPV types 11,16,18,31,33,35,45, 52,58 , and antibodies against Chlamydia trachomatis and Human herpesvirus 2. Multivariate analyses were performed to estimate hazard ratios (HR), odds ratios (OR) and corresponding $95 \%$ confidence intervals $(\mathrm{Cl})$. The cohort analysis showed that number of fullterm pregnancies was positively associated with $\mathrm{CIN} 3 / \mathrm{CIS}$ risk ( $p$-trend $=0.03$ ). Duration of oral contraceptives use was associated with a significantly increased risk of both $\mathrm{CIN} 3 / \mathrm{CIS}$ and ICC ( $\mathrm{HR}=1.6$ and $\mathrm{HR}=1.8$ respectively for $\geq 15$ years versus never use). Ever use of menopausal hormone therapy was associated with a reduced risk of ICC (HR $=0.5,95 \%$ $\mathrm{Cl}$ : 0.4-0.8). A non-significant reduced risk of ICC with ever use of intrauterine devices (IUD) was found in the nested case-control analysis $(\mathrm{OR}=0.6)$. Analyses restricted to all cases and HPV seropositive controls yielded similar results, revealing a significant inverse association with IUD for combined CIN3/CIS and ICC (OR $=0.7)$.

\section{Conclusions}

Even though HPV is the necessary cause of $\mathrm{CC}$, our results suggest that several hormonal factors are risk factors for cervical carcinogenesis. Adherence to current cervical cancer screening guidelines should minimize the increased risk of $\mathrm{CC}$ associated with these hormonal risk factors. 


\section{Introduction}

Cervical cancer (CC) is the fourth most common cancer among women worldwide with an estimated 528,000 new cases and the fourth most common cause of female death from cancer with an estimated 266,000 deaths in 2012 [1]. Human papillomavirus (HPV) is one of the most common sexually transmitted infections worldwide. In fact, most sexually active women will be infected with HPV during their lifetime, although the majority of HPV infections are cleared within 2 years [2,3]. HPV genotypes are classified as low-risk or high-risk based on their association with cervical cancer (CC) [4]. It is well established that persistent infection with high-risk HPV genotypes is the necessary although not sufficient cause of CC [5]. Thus, the involvement of other factors, in addition to HPV, is needed to induce cervical carcinogenesis. High parity and hormonal contraceptives have long been recognized as potential cofactors of CC [5]. A comprehensive review conducted by the International Agency for Research on Cancer (IARC) classified the use of combined oral contraceptives (OC) as carcinogenic to humans, and this was partly based on the reported associations with CC [6]. A collaborative pooled reanalysis evaluating CC, hormonal contraceptives and parity found an increased risk of CC in current and long-term OC users, a reduced risk after stopping these hormones [7], and positive associations with both number of full-term pregnancies (FTP) and an early age at first FTP [8]. In addition, results from the European Prospective Investigation into Cancer and Nutrition (EPIC) showed that circulating levels of sex steroid hormones testosterone and possibly estradiol were also positively involved in the etiology of CC [9]. However, even though these associations are generally consistent across studies, it must be noted that the evidence for a role of hormones in cervical carcinogenesis is mostly derived from retrospective case-control studies that did not always take into account HPV. Thus the aim of this study is to prospectively examine potential associations between hormonal factors and risk of developing cervical cancer and pre-cancer using data from a large prospective study that additionally uses serological markers of HPV exposure.

\section{Materials and Methods The EPIC cohort study}

The EPIC study is a large prospective cohort study including 521,448 participants (367,993 women and 153,455 men) recruited between 1992 and 2000 through 23 centres in 10 European countries: Denmark, France, Germany, Greece, Italy, the Netherlands, Norway, Spain, Sweden, and the United Kingdom. Most of the EPIC participants were between the ages of 35 and 70 years. The study procedures have been described in detail elsewhere $[9,10]$. At recruitment, participants gave their written informed consent and completed questionnaires on their diet, medical and lifestyle history. They were also invited to provide blood samples for future testing of markers of interest. The EPIC study was approved by the ethical review committees from each center.

\section{Study population}

Of the approximately 370,000 women enrolled in the study, women were not eligible for this analysis if they had prevalent cancer or pre-cancer $(n=22,180)$, incomplete follow-up $(\mathrm{n}=2,295)$, hysterectomy $(\mathrm{n}=34,973)$ or incomplete lifestyle questionnaire $(\mathrm{n}=509)$ at baseline. This left a total of 308,036 women in these analyses.

\section{Identification of cases and follow-up}

Cases of cervical intraepithelial neoplasia grade 3 (CIN3)/carcinoma in situ (CIS) and invasive cervical cancer (ICC) were identified through several methods, including a record linkage with population-based cancer registries (Denmark, Italy, the Netherlands, Norway, Spain, Sweden 
and the United Kingdom), health insurance records, hospital-based cancer and pathology registries and active follow-up of subjects (France, Germany and Greece). Data on vital status were obtained from mortality registries at regional and national level. Cervical cancer cases included only those women with first primary incident cancer according to the International Classification of Diseases, 10th revision (code C53: cervix uteri). Contrary to ICC, ascertainment of CIN3/CIS cases was not systematically done in all cancer registries and EPIC centers. Follow-up time was calculated between the date at recruitment and the date at diagnosis for cases or the date at censoring (death, loss of follow-up or end of follow-up) for non-cases. The end of follow-up ranged from December 2003 to December 2006, depending on the center. The median follow-up time in this cohort was around 9 years $\left(25^{\text {th }}-75^{\text {th }}\right.$ percentile: $7.5-10.8$ years $)$ contributing a total of $2,775,235$ person-year. Among the 308,036 women included in the final analysis 1,065 cases were identified: 261 ICC cases and 804 CIN3/CIS cases. Detailed tumor histology was specified for 953 cases $(89 \%)$, of which 901 (95\%) were classified as squamous cell carcinoma (SCC, 712 in situ and 189 invasive) and 52 (5\%) as adenocarcinoma (9 in situ and 43 invasive).

\section{Nested case-control study}

A nested case-control study within the EPIC cohort was conducted to allow for the adjustment by serological markers of HPV and other sexually transmitted infections (STIs), even though this strategy involves a lower number of subjects. For each case with available blood sample, two matched control subjects were randomly selected from the cancer-free cohort of women that were at risk at the time of diagnosis of the corresponding case. Matching criteria included: study center, age at recruitment (5 year intervals), menopausal status (pre-, peri- and postmenopausal), follow-up time, date, time and fasting status at blood collection, and, among premenopausal women, phase of the menstrual cycle [9]. Approximately $70 \%$ of ICC cases and $53 \%$ of CIN3/CIS cases identified in the cohort provided serum samples, yielding a total of 609 cases (184 ICC and 425 CIN3/CIS) and 1,218 controls for the analyses.

\section{Serological testing}

HPV serology was performed at the German Cancer Research Centre in Heidelberg, Germany. Assay procedures have been explained in detail previously $[11,12]$. Briefly, antibodies to the capsid protein L1 of high-risk mucosal HPV types 16, 18, 31, 33, 35, 45, 52, and 58, and of lowrisk mucosal HPV type 11 were tested by Glutathione S-transferase capture and fluorescent bead-based multiplex serology [13-15]. For the nested case-control analyses, HPV L1 seropositivity refers to that for at least one of the nine HPV types analyzed, and high-risk HPV L1 seropositivity refers to positivity for at least one of the eight high-risk HPV types analyzed.

Serum antibodies against Chlamydia trachomatis (CT) and Human herpesvirus 2 (HHV-2) were tested at the Hospital of Santa Creu i Sant Pau in Barcelona, Spain. We used the commercial assay Chlamydia MIF IgG (Focus Diagnostics, Cypress, CA, USA) for the detection of CT IgG serum antibodies performed by microimmunofluorescence, and the commercial kit HerpeSelect $\AA 2$ ELISA IgG (Focus Diagnostics, Cypress, CA, USA) for the detection of antibodies against HHV-2 evaluated by an enzyme immunoassay. The positive results of HHV-2 antibodies were confirmed by a membrane-based immunoassay with the biokitHSV-2 Rapid Test (Biokit USA, Lexington, MA, USA).

All serological assays were performed blinded to the subject's characteristics.

\section{Statistical analyses}

Multivariate hazard ratios (HR) and 95\% confidence intervals (CI) were estimated to evaluate the risk of CIN3/CIS and ICC in relation to several hormonal risk factors using Cox 
proportional hazard regression models. In all analyses, age was used as the underlying time variable, and the models were stratified by age at recruitment (in one-year categories) and study center. The proportional hazards assumption was evaluated for all models fitted using tests based on weighted residuals. Tests for linear trend were performed using continuous variables.

We estimated HR for variables related with hormonal factors collected at recruitment. The parity-related variables evaluated in these analyses were the following: ever FTP (never, ever), number of FTP $(1,2,3, \geq 4)$, age at first FTP $(\geq 30,25-29,21-24$, $\leq 20$ years old), and number of induced abortions $(1, \geq 2)$ if any (never, ever). Self-reported baseline menopausal status was defined as postmenopausal (no menses in the last 12 months or bilateral ovariectomy), perimenopausal ( $<9$ menses in the past 12 months), and premenopausal (regular menses in the past 12 months). Women with unknown menopausal status were classified as postmenopausal if they were 55 years old or more, perimenopausal if they were between 46 and 55 years of age and premenopausal when they were less than 46 years of age at recruitment. We estimated the cumulative years of menstrual cycling as the difference between the age at menopause (for postmenopausal women) or of the age at recruitment (for pre- and perimenopausal women) and the age at menarche minus the total time being pregnant (number of FTP $x 9$ months) and the total time using OCs; this variable was categorized in quintiles: $\leq 19.50,19.51-26.76$, $26.77-31.50,31.51-35.50, \geq 35.51$ years. The exogenous hormone-related factors evaluated included: OC use (never, ever, past, current), duration of OC use $(\leq 1,2-4,5-9,10-14, \geq 15$ years), latency of OC use or time since first use ( $\leq 10,11-20, \geq 21$ years), and recency of OC use or time since last use $(\leq 5,6-14, \geq 15$ years) among past OC users. Use of intrauterine device (IUD) was also analyzed and dichotomized as never and ever IUD use. Among postand perimenopausal women, use of menopausal hormone therapy (HT) was evaluated as: HT use (never, ever, past, current), duration of HT use ( $\leq 1,2-4, \geq 5$ years) and HT formulation (estrogen alone, progesterone alone, combination of estrogen/progesterone). Ovariectomy was also assessed (no, unilateral, bilateral). Combined variables using number of FTP and age at first FTP, duration and recency of OC use, and number of FTP and duration of OC use were also created and evaluated. The following variables were also analyzed but not included in the tables presented because they were either collinear with the final selected variables or not associated with risk in any of the analyses: time since first FTP, pregnancies, live births, stillbirths, miscarriages, breastfeeding, age started and stopped OC use, use of different contraceptives (condoms, spermicidal creams, tubal ligation, rhythm methods, diaphragm, vasectomy), age started HT use, type of HT (oral, injectable, topical), menopausal status, age at menarche, and age at menopause.

We used stepwise regression modeling to assess potential confounding by other variables such as: body mass index (BMI, underweight ( $<18.5$ ), normal (18.5-25), overweight (25-30), obese ( $\geq 30)$ ), marital status (single, married/cohabiting, divorced/separated, widowed), education level (none, primary school, technical/professional school, secondary school, university degree), physical activity (inactive, moderately inactive, moderately active, and active, using a validated Cambridge Physical Activity Index [16]), and smoking habits (never, former and duration $<15$ years, former and duration $\geq 15$ years, current and intensity $<10 \mathrm{cig} /$ day, current and intensity $\geq 10 \mathrm{cig} /$ day). Number of FTP $(0,1,2,3, \geq 4)$, OC use and duration (never, past for $<10$ years, past for $\geq 10$ years, current for $<10$ years, current for $\geq 10$ years), and menopausal status with HT use (premenopausal, peri- and postmenopausal and non HT users, periand postmenopausal and HT users) were used as adjusting variables when appropriate.

In the nested case-control analysis, multivariate odds ratios (OR) and 95\% CI were estimated to evaluate the risk of CIN3/CIS and ICC in relation to hormonal factors using conditional logistic regression models. Models were adjusted for HPV L1 serology, CT serology, HHV-2 serology, and other potential confounding factors (BMI, marital status, education 
level, physical activity, smoking habits, OC use and duration, number of FTP and menopausal status with HT use (when appropriate)). Unconditional logistic regression analyses among all cases and HPV L1 seropositive controls were also performed, including the matching variables in the models as adjusting covariates. Tests for interaction among hormonal variables and other risk factors were based on the likelihood ratio test comparing the models with and without the interaction terms.

When applicable, variables included a missing or unknown category in order to avoid the exclusion of participants in the regression models. All statistical tests were 2-tailed, and p-values below 0.05 were considered statistically significant. Statistical analyses were performed using the R programing language (R Development Core Team, 2005, http://www.R-project.org).

\section{Ethics statement}

All participants gave written informed consent, and the study was approved by the local ethics committees in the participating countries (Athens: University of Athens Medical School; Cambridge: Norwich District Ethics Committee; Denmark (Aarhus, Copenhagen): The National Committee on Health Research Ethics; France (Paris): Comité de Protection des Personnes; Heidelberg: Ethics Committee of the Heidelberg University Medical School; International Agency for Research on Cancer: IARC Ethics Committee; Imperial College: Imperial College Research Ethics Committee [ICREC]; Italy (Florence, Milan, Naples, Ragusa, Turin): Comitato Etico Indipendente, Fondazione IRCCS Istituto Nazionale dei Tumori, Milano; Florence: Comitato Etico Locale Azienda Sanitaria di Firenze; Malmo: Ethics Committee of Lundst University; Netherlands (Bilthoven and Utrecht): The Medical Ethical Committee (METC = Medisch Ethische Toetsingscommissie) of the University Medical Center Utrecht (UMCU), Utrecht, the Netherlands; Norway: Regional ethical committee for Northern Norway and the Norwegian Data Inspectorate; Oxford: Scotland A Research Ethics Committee; Potsdam: Ethikkommission der Landesärztekammer Brandenburg Cottbus, Deutschland; Spain (Asturias, Barcelona, Granada, Murcia, Navarre, San Sebastian): CEIC Comité de Ética de Investigación Clínica; Turin: Human Genetics Foundation Torino: Ethics Committee; Umea: Umea Regional Ethical Review Board) and the Internal Review Board of the International Agency for Research on Cancer.

\section{Results}

Baseline characteristics for cases and non-cases included in the cohort analysis have been reported previously (S1 Table) [11]. In brief, most CIN3/CIS cases were recruited from the United Kingdom (37.1\%), Sweden (20.8\%) and Norway (15.0\%), and most of the ICC cases were from Sweden (16.9\%), the United Kingdom (13.4\%), Denmark (12.6\%) and France (11.5\%). CIN3/CIS cases were younger than ICC cases. As compared to non-cases, women with CIN3/CIS or ICC were more likely to be single or separated, to smoke, to have ever used OCs, and to be premenopausal. The characteristics of the women included in the nested casecontrol analysis have already been described in two previous articles [11,12].

Table 1 shows associations between factors related to endogenous hormones and risk of developing CIN3/CIS and ICC by study design (full cohort study and nested case-control study). In both analyses the risk of CIN3/CIS increased significantly with increasing number of FTP. In contrast, for ICC, a non significant decreased risk was observed among women who ever had a FTP. A decreased risk of CIN3/CIS and ICC was observed with decreasing age at first FTP, but only in the case-control study and this was only statistically significant for CIN3/ CIS. In both study designs, a non significant decreased risk of CIN3/CIS was observed among women who had more than one induced abortion. However, for ICC an increased risk was 
Table 1. Risk of CIN3/CIS and ICC of the cervix according to factors related with endogenous hormones.

\begin{tabular}{|c|c|c|c|c|c|c|c|c|}
\hline \multirow[t]{3}{*}{ Risk factor } & \multicolumn{4}{|c|}{ Cohort study } & \multicolumn{4}{|c|}{ Nested case-control study } \\
\hline & \multicolumn{2}{|c|}{ CIN3/CIS } & \multicolumn{2}{|l|}{ ICC } & \multicolumn{2}{|c|}{ CIN3/CIS } & \multicolumn{2}{|c|}{ ICC } \\
\hline & Non-cases / Cases & $\begin{array}{c}\text { HR } \\
(95 \% \text { Cl) }\end{array}$ & Non-cases / Cases & $\begin{array}{c}\text { HR } \\
(95 \% \text { Cl) }\end{array}$ & Controls / Cases & $\begin{array}{c}\text { OR } \\
(95 \% \mathrm{Cl})^{2}\end{array}$ & Controls / Cases & $\begin{array}{c}\text { OR } \\
(95 \% \mathrm{Cl})^{2}\end{array}$ \\
\hline \multicolumn{9}{|l|}{ Number of FTP } \\
\hline Never & 45,228 / 191 & 1.0 (ref) & $45,419 / 45$ & 1.0 (ref) & $139 / 51$ & 1.0 (ref) & $43 / 30$ & 1.0 (ref) \\
\hline Ever & $246,823 / 516$ & $1.5(1.2-1.9)$ & $247,339 / 191$ & $0.7(0.5-1.0)$ & $543 / 288$ & $2.0(1.2-3.2)$ & $281 / 131$ & $0.8(0.4-1.5)$ \\
\hline 1 & $44,007 / 116$ & $1.4(1.0-1.8)$ & $44,123 / 36$ & $0.7(0.5-1.2)$ & $132 / 71$ & $1.7(1.0-2.9)$ & 42 / 26 & $0.8(0.3-2.0)$ \\
\hline 2 & $114,956 / 237$ & $1.4(1.1-1.9)$ & $115,193 / 82$ & $0.7(0.4-1.0)$ & $247 / 132$ & $2.1(1.2-3.4)$ & $153 / 53$ & $0.5(0.2-1.1)$ \\
\hline 3 & $54,107 / 94$ & $1.4(1.0-1.9)$ & $54,201 / 40$ & $0.7(0.4-1.2)$ & $97 / 46$ & $2.1(1.2-3.8)$ & $55 / 28$ & $1.0(0.4-2.3)$ \\
\hline$\geq 4$ & $23,545 / 51$ & $2.3(1.6-3.3)$ & $23,596 / 23$ & $0.9(0.5-1.6)$ & $42 / 28$ & $2.6(1.3-5.3)$ & $18 / 16$ & $1.5(0.5-4.4)$ \\
\hline $\begin{array}{l}\text { p for trend among ever } \\
\text { ftp }\end{array}$ & & 0.03 & & 0.4 & & 0.2 & & 0.02 \\
\hline \multicolumn{9}{|l|}{$\begin{array}{l}\text { Age at first FTP } \\
\text { (years) }{ }^{3}\end{array}$} \\
\hline$\geq 30$ & $35,122 / 71$ & 1.0 (ref) & $35,193 / 22$ & 1.0 (ref) & $69 / 39$ & 1.0 (ref) & $22 / 13$ & 1.0 (ref) \\
\hline $25-29$ & $85,567 / 161$ & $1.1(0.8-1.4)$ & 85,728 / 66 & $1.2(0.7-2.0)$ & 164 / 90 & $1.0(0.6-1.8)$ & $104 / 48$ & $0.8(0.2-2.9)$ \\
\hline $21-24$ & $89,750 / 181$ & $1.1(0.8-1.5)$ & $89,931 / 65$ & $1.0(0.6-1.6)$ & $192 / 106$ & $0.7(0.4-1.3)$ & $107 / 41$ & $0.6(0.2-1.9)$ \\
\hline$\leq 20$ & 35,372 / 101 & $1.2(0.9-1.7)$ & $35,473 / 36$ & $1.1(0.6-2.0)$ & $113 / 52$ & $0.5(0.3-1.1)$ & $47 / 27$ & $1.0(0.2-4.3)$ \\
\hline $\begin{array}{l}\text { p for trend among ever } \\
\text { ftp }\end{array}$ & & 0.2 & & 0.8 & & 0.03 & & 0.9 \\
\hline \multicolumn{9}{|l|}{$\begin{array}{l}\text { Number of induced } \\
\text { abortions }\end{array}$} \\
\hline Never & $157,566 / 278$ & 1.0 (ref) & 157,844 / 99 & 1.0 (ref) & $295 / 166$ & 1.0 (ref) & $180 / 70$ & 1.0 (ref) \\
\hline Ever & $41,079 / 77$ & $0.9(0.7-1.1)$ & $41,156 / 53$ & $1.9(1.3-2.7)$ & 84 / 45 & $0.6(0.4-1.0)$ & $57 / 41$ & $1.7(0.8-3.4)$ \\
\hline 1 & $28,058 / 63$ & $1.0(0.7-1.3)$ & $28,121 / 36$ & $1.9(1.3-2.8)$ & $63 / 36$ & $0.6(0.4-1.2)$ & $38 / 26$ & $1.4(0.6-3.4)$ \\
\hline$\geq 2$ & $12,722 / 13$ & $0.6(0.3-1.0)$ & $12,735 / 17$ & $1.8(1.0-3.2)$ & $21 / 9$ & $0.5(0.2-1.2)$ & $18 / 15$ & $2.1(0.8-6.1)$ \\
\hline \multicolumn{9}{|l|}{$\begin{array}{l}\text { Cumulative years of } \\
\text { menstrual cycles } \\
\text { without OCs } \\
\text { (quintiles) }^{5}\end{array}$} \\
\hline Quintile 1 & 47,020 / 270 & 1.0 (ref) & $47,290 / 57$ & 1.0 (ref) & $126 / 78$ & 1.0 (ref) & $43 / 26$ & 1.0 (ref) \\
\hline Quintile 2 & $46,953 / 118$ & $0.9(0.7-1.1)$ & $47,071 / 29$ & $0.5(0.3-0.8)$ & $124 / 64$ & $1.0(0.6-1.7)$ & 39 / 28 & $1.6(0.6-3.8)$ \\
\hline Quintile 3 & $48,557 / 91$ & $0.8(0.6-1.1)$ & $48,648 / 49$ & $0.8(0.5-1.3)$ & $106 / 57$ & $0.8(0.5-1.4)$ & $66 / 22$ & $0.5(0.2-1.2)$ \\
\hline Quintile 4 & 47,855 / 63 & $0.7(0.5-1.0)$ & 47,918 / 36 & $0.6(0.4-1.1)$ & $111 / 43$ & $0.5(0.3-0.9)$ & $60 / 39$ & $1.0(0.4-2.3)$ \\
\hline Quintile 5 & $44,733 / 47$ & $0.7(0.5-1.1)$ & 44,780 / 24 & $0.4(0.2-0.8)$ & 114 / 49 & $0.5(0.3-0.9)$ & $74 / 23$ & $0.7(0.3-1.7)$ \\
\hline$p$ for trend & & 0.009 & & 0.005 & & 0.01 & & 0.6 \\
\hline
\end{tabular}

CIN3: cervical intraepithelial neoplasia grade 3; CIS: carcinoma in situ; ICC: invasive cervical cancer; HR: hazard ratio; OR: odds ratio; Cl: confidence interval; FTP: full-term pregnancy; OC: oral contraceptives; HT: hormone therapy. The number of cases does not add up the total number of cases because of missing values. Bold font indicates a statistically significant effect $(p<0.05)$.

${ }^{1}$ Models were adjusted by body mass index, marital status, level education, physical activity, smoking habits, OC use and duration and menopausal status with HT use.

${ }^{2}$ Conditional regression models were adjusted by HPV L1 serology, Chlamydia trachomatis serology, Human herpesvirus 2 serology, body mass index, marital status, level education, physical activity, smoking habits, OC use and duration and menopausal status with HT use. See methods for list matching variables.

${ }^{3}$ Among parous women.

${ }^{4}$ Excludes Bilthoven, Sweden and Norway because information was not collected for this variable.

${ }^{5}$ Model not adjusted by OC use and duration because of co-linearity with cumulative duration of menstrual cycles. For cohort study, quintiles correspond to: q1: $\leq 19.50$; q2: 19.51-26.76; q3: 26.77-31.50; q4: 31.51-35.50; q5: $\geq 35.51$. For nested case-control study, quintiles correspond to: q1: $\leq 14.66$; q2: 14.67-24.23; q3: 24.24-29.17; q4: 29.18-33.75; q5: $\geq 33.76$.

doi:10.1371/journal.pone.0147029.t001 
found among women who had at least one induced abortion but the association was statistically significant only in the cohort study. When analyses were adjusted or stratified by number of FTP, the magnitude of the point estimates of the associations with both outcomes remained mostly unchanged (data not shown). Women with higher lifetime years of menstrual cycles had a lower risk of CIN3/CIS in both studies and of ICC in the cohort study.

When analyses were restricted to parous women and mutually adjusted for number of FTP and age at first FTP, the effect of number of FTP for CIN3/CIS risk was maintained in the full cohort analysis ( $\mathrm{HR}=1.6,95 \% \mathrm{CI}: 1.1-2.3)$, and was of borderline statistical significance in the nested case-control study ( $\mathrm{OR}=2.1,95 \% \mathrm{CI}$ : $1.0-4.6$; data not shown). No associations were found for ICC risk.

Table 2 summarizes associations with factors related to exogenous hormones. Current use of OCs and increasing years of use were both associated with CIN3/CIS and ICC in the cohort study, even though the trend was only statistically significant for CIN3/CIS. As compared to current users, increasing years since last OC use was associated with a reduction in the risk of developing CIN3/CIS in the cohort study. In the case-control study associations were found in the same direction but did not reach statistical significance. Ever use of HT among peri- and postmenopausal women significantly decreased the risk of ICC in both studies. We found a decreased risk of ICC with duration of HT use but the trend did not reach statistical significance. In contrast, for CIN3/CIS we found a statistically significant reduced risk with years of HT in the case-control study. Similar results were obtained when analyses where restricted to postmenopausal women only (data not shown). We also assessed the effect of HT formulation finding a non significant increased risk of CIN3/CIS for users of menopausal estrogens alone (HR $=1.7,95 \%$ CI: 0.9-3.1, for ever versus never users) and a lack of association for combined formulations (data not shown). The effect of progestin alone could not be assessed due to the low number of exposed subjects. For ICC, most exposed cases used some kind of combination of hormones showing a borderline inverse association ( $\mathrm{HR}=0.6,95 \% \mathrm{CI}$ : 0.3-1.0, for ever versus never users). There were not enough cases to assess the effect of estrogen or progestin alone (data not shown). Concerning IUD use, a non significant inverse association with both CIN3/ CIS and ICC risk was observed in the nested case-control study.

When the combined effect of duration and recency of OC use was evaluated, the risk of CIN3/CIS declined progressively with increasing years since last use (data not shown). This pattern was not observed for ICC risk.

In both study designs, the combined effect of number of FTP and duration of OC use was analyzed, and showed a significant increased risk of CIN3/CIS with increasing number of FTP within each category of OC use (S2 Table). Women with 4 or more FTP had a four-fold risk within each category of $\mathrm{OC}$ use as compared to women who were nulliparous and never used OCs. The test of interaction between OC use and FTP reached statistical significance in the cohort study $(p=0.004)$. In contrast, for ICC we only found a marginal increased risk among multiparous women who used OCs for more than 5 years in the cohort study.

Concerning associations by histological type, the risk of SCC showed the same overall pattern: increased risk of CIS with number of FTP and years of OC use and decreased risk with years of HT use, and increased risk of invasive SCC with years of OC use and decreased risk with years of HT use (data not shown). Regarding adenocarcinomas, associations could not be evaluated accurately because of the small number of cases ( 52 cases in the cohort study and 33 cases in the nested case-control study for both in situ and invasive adenocarcinomas). Nevertheless, these analyses showed non significant and weak positive associations with number of FTP and OC use for invasive adenocarcinoma, and a non significant inverse association with HT use (data not shown). 
Table 2. Risk of CIN3/CIS and ICC of the cervix according to factors related with exogenous hormones.

\begin{tabular}{|c|c|c|c|c|c|c|c|c|}
\hline \multirow[t]{3}{*}{ Risk factor } & \multicolumn{4}{|c|}{ Cohort study } & \multicolumn{4}{|c|}{ Nested case-control study } \\
\hline & \multicolumn{2}{|c|}{ CIN3/CIS } & \multicolumn{2}{|c|}{ ICC } & \multicolumn{2}{|c|}{ CIN3/CIS } & \multicolumn{2}{|c|}{ ICC } \\
\hline & Non-cases / Cases & $\mathrm{HR}(95 \% \mathrm{Cl})^{1}$ & Non-cases / Cases & $\mathrm{HR}(95 \% \mathrm{Cl})^{1}$ & Controls / Cases & OR $(95 \% \mathrm{Cl})^{2}$ & Controls / Cases & OR $(95 \% \mathrm{Cl})^{2}$ \\
\hline \multicolumn{9}{|l|}{ IUD use $^{3}$} \\
\hline Never & $170,843 / 371$ & 1.0 (ref) & $171,214 / 144$ & 1.0 (ref) & $325 / 160$ & 1.0 (ref) & $194 / 106$ & 1.0 (ref) \\
\hline Ever & $63,677 / 136$ & $1.1(0.9-1.4)$ & $63,813 / 43$ & $0.9(0.6-1.3)$ & $160 / 82$ & $0.8(0.5-1.3)$ & $70 / 28$ & $0.6(0.3-1.2)$ \\
\hline \multicolumn{9}{|l|}{ OC use } \\
\hline Never & $121,117 / 169$ & 1.0 (ref) & $121,286 / 76$ & 1.0 (ref) & $225 / 99$ & 1.0 (ref) & $139 / 56$ & 1.0 (ref) \\
\hline Ever & $176,993 / 548$ & $1.1(0.9-1.3)$ & $177,541 / 165$ & $1.6(1.1-2.3)$ & $466 / 244$ & $1.1(0.8-1.5)$ & $186 / 109$ & $1.5(0.8-2.6)$ \\
\hline Past & $152,658 / 411$ & $1.0(0.9-1.3)$ & $153,069 / 134$ & $1.6(1.1-2.2)$ & $392 / 197$ & $1.0(0.7-1.5)$ & $158 / 86$ & $1.3(0.7-2.4)$ \\
\hline Current & 17,384 / 127 & $1.8(1.4-2.4)$ & $17,511 / 22$ & $2.2(1.3-4.0)$ & $57 / 41$ & $1.7(0.9-3.1)$ & $24 / 17$ & $2.2(0.7-6.7)$ \\
\hline \multicolumn{9}{|l|}{$\begin{array}{l}\text { Duration of } \mathrm{OC} \text { use } \\
\text { (years) }\end{array}$} \\
\hline Never & $121,117 / 169$ & 1.0 (ref) & $121,286 / 76$ & 1.0 (ref) & $225 / 99$ & 1.0 (ref) & $139 / 56$ & 1.0 (ref) \\
\hline$\leq 1$ & $31,867 / 78$ & $1.0(0.8-1.3)$ & $31,945 / 27$ & $1.5(0.9-2.4)$ & \multirow[t]{2}{*}{$180 / 82$} & \multirow[t]{2}{*}{$1.0(0.6-1.5)$} & \multirow[t]{2}{*}{$76 / 36$} & \multirow[t]{2}{*}{$1.1(0.6-2.2)$} \\
\hline $2-4$ & $40,168 / 127$ & $1.1(0.8-1.4)$ & $40,295 / 27$ & $1.3(0.8-2.0)$ & & & & \\
\hline $5-9$ & $38,816 / 136$ & $1.1(0.9-1.4)$ & $38,952 / 41$ & $2.0(1.3-3.0)$ & $108 / 54$ & $1.1(0.7-1.7)$ & $38 / 22$ & $1.6(0.7-3.7)$ \\
\hline $10-14$ & 26,969 / 90 & $1.2(0.9-1.6)$ & 27,059 / 26 & $1.6(1.0-2.6)$ & \multirow[t]{2}{*}{$138 / 94$} & \multirow[t]{2}{*}{$1.6(1.0-2.5)$} & \multirow[t]{2}{*}{$63 / 42$} & \multirow[t]{2}{*}{$1.5(0.7-3.1)$} \\
\hline$\geq 15$ & $23,395 / 82$ & $1.6(1.2-2.2)$ & $23,477 / 28$ & $1.8(1.1-2.9)$ & & & & \\
\hline $\begin{array}{l}p \text { for trend among } \mathrm{OC} \\
\text { users }\end{array}$ & & 0.01 & & 0.2 & & 0.2 & & 0.9 \\
\hline \multicolumn{9}{|l|}{$\begin{array}{l}\text { Recency of OC use } \\
\text { (years) }{ }^{4,5}\end{array}$} \\
\hline Current & $7,678 / 95$ & 1.0 (ref) & $7,773 / 8$ & 1.0 (ref) & $32 / 20$ & 1.0 (ref) & \multirow[t]{2}{*}{$10 / 12$} & \multirow[t]{2}{*}{1.0 (ref) $^{7}$} \\
\hline$\leq 5$ & $9,662 / 79$ & $0.7(0.5-1.0)$ & $9,741 / 11$ & $1.1(0.4-2.8)$ & $30 / 21$ & $1.0(0.2-4.2)$ & & \\
\hline $6-14$ & $17,368 / 60$ & $0.6(0.4-0.8)$ & $17,428 / 14$ & $0.8(0.3-2.0)$ & $44 / 21$ & $0.4(0.1-1.9)$ & $27 / 11$ & - \\
\hline$\geq 15$ & $25,681 / 45$ & $0.6(0.4-0.9)$ & $25,726 / 25$ & $1.0(0.4-2.5)$ & $48 / 22$ & $0.6(0.1-5.0)$ & $32 / 20$ & - \\
\hline $\begin{array}{l}p \text { for trend among past OC } \\
\text { users }\end{array}$ & & 0.2 & & 0.7 & & - & & - \\
\hline \multicolumn{9}{|l|}{ HT use ${ }^{6}$} \\
\hline Never & $114,271 / 149$ & 1.0 (ref) & $114,420 / 94$ & 1.0 (ref) & $170 / 83$ & 1.0 (ref) & $107 / 67$ & 1.0 (ref) \\
\hline Ever & $63,839 / 131$ & $1.2(0.9-1.5)$ & $63,970 / 31$ & $0.5(0.4-0.8)$ & $149 / 84$ & $0.9(0.6-1.5)$ & $72 / 18$ & $0.3(0.1-0.7)$ \\
\hline Past & $18,508 / 22$ & $1.0(0.6-1.5)$ & $18,530 / 11$ & $0.6(0.3-1.2)$ & $42 / 17$ & $0.7(0.3-1.4)$ & $19 / 8$ & $0.6(0.2-2.0)$ \\
\hline Current & $43,110 / 102$ & $1.3(1.0-1.7)$ & $43,212 / 19$ & $0.5(0.3-0.8)$ & 102 / 61 & $1.1(0.7-1.9)$ & $51 / 10$ & $0.2(0.1-0.5)$ \\
\hline \multicolumn{9}{|l|}{$\begin{array}{l}\text { Duration of HT use } \\
\text { (years) }{ }^{6}\end{array}$} \\
\hline Never & $114,271 / 149$ & 1.0 (ref) & $114,420 / 94$ & 1.0 (ref) & $170 / 83$ & 1.0 (ref) & $107 / 67$ & 1.0 (ref) \\
\hline$\leq 1$ & $22,819 / 43$ & $1.3(0.9-1.9)$ & $22,862 / 12$ & $0.7(0.4-1.2)$ & $42 / 28$ & $1.2(0.6-2.5)$ & \multirow[t]{2}{*}{$39 / 11$} & \multirow[t]{2}{*}{$0.4(0.1-1.0)$} \\
\hline $2-4$ & 19,032 / 32 & $1.0(0.7-1.5)$ & 19,064 / 9 & $0.6(0.3-1.1)$ & $44 / 15$ & $0.5(0.2-1.1)$ & & \\
\hline$\geq 5$ & $15,834 / 27$ & $1.0(0.7-1.6)$ & $15,861 / 7$ & $0.4(0.2-0.9)$ & $49 / 19$ & $0.6(0.3-1.4)$ & $26 / 5$ & $0.1(0.03-0.6)$ \\
\hline $\begin{array}{l}\text { p for trend among } \\
\text { menopausal hormones } \\
\text { users }\end{array}$ & & 0.2 & & 0.4 & & - & & - \\
\hline
\end{tabular}

CIN 3: cervical intraepithelial neoplasia grade 3; CIS: carcinoma in situ; ICC: invasive cervical cancer; HR: hazard ratio; OR: odds ratio; CI: confidence interval; IUD: intrauterine device; OC: oral contraceptives; HT: hormone therapy. The number of cases does not add up the total number of cases because of missing values. Bold font indicates a statistically significant effect $(p<0.05)$.

${ }^{1}$ Models were adjusted by body mass index, marital status, level education, physical activity, smoking habits, number of full-term pregnancies and menopausal status with HT use.

${ }^{2}$ Conditional regression models were adjusted by HPV L1 serology, Chlamydia trachomatis serology, Human herpesvirus 2 serology, body mass index, marital status, level education, physical activity, smoking habits, number of full-term pregnancies and menopausal status with HT use. See methods for list matching variables.

${ }^{3}$ Excludes Bilthoven, Sweden and Norway because information was not collected for this variable.

${ }^{4}$ Among OC users (excluding non OC users).

${ }^{5}$ Excludes Bilthoven, France, Germany, Sweden, Denmark and Norway because information was not collected for this variable.

${ }^{6}$ Among peri and postmenopausal women (excluding premenopausal women) and models also adjusted by OC use and duration but not adjusted by menopausal status with HT use. ${ }^{7}$ Risk estimates were not estimated due to lack of power in the model.

doi:10.1371/journal.pone.0147029.t002 
Analyses restricted to countries with screening programs (United Kingdom, Sweden, Denmark, Norway) did not substantially change the magnitude of the associations for the several risk factors (data not shown).

Table 3 shows associations restricted to all cases and HPV L1 seropositive controls in the nested case-control study. In this analysis number of FTP was strongly associated with an increased risk of CIN3/CIS. For induced abortions we found opposite effects for CIN3/CIS versus ICC ( $\mathrm{OR}=0.5$ and $\mathrm{OR}=1.7$ respectively, for ever versus never). Current use and duration of OCs increased the risk of both CIN3/CIS and ICC although the associations were not statistically significant. HT use and duration was inversely and significantly associated with ICC. Finally, IUD use was inversely associated with both CIN3/CIS and ICC risk without statistical significance, although when we combined CIN3/CIS and ICC the significance emerged $(\mathrm{OR}=0.7,95 \%$ CI: 0.5-0.96; data not shown). Associations were broadly similar in analyses restricted to all cases and high-risk HPV seropositive controls and in analyses including only HPV seropositive cases and controls (data not shown), revealing a significant inverse association between IUD use and ICC among HPV seropositive women (OR = 0.3, 95\% CI: 0.1-0.9; data not shown).

\section{Discussion}

The results of this large prospective cohort study show that certain endogenous and exogenous hormonal factors appear to be related to cervical carcinogenesis. Thus the risk of cervical precancer increased with increasing number of FTP and duration of OC use, and decreased with increasing years since last OC use among past users. For ICC, the risk increased with number of abortions and duration of OC use, and decreased with increasing duration of HT. A reduced risk of ICC was also observed among IUD users in the nested case-control study. Globally the associations were somewhat stronger in the cohort study than in the nested case-control study. The case-control study is useful to support the results obtained in the cohort study since it allowed for the additional adjustment of serological markers of HPV exposure and other STIs.

\section{OC use}

Consistent with results from previously published pooled analyses [7,17], our study highlighted strong and positive associations between OC use and risk of cervical cancer and pre-cancer; specifically, the risk increased with duration of use and decreased with cessation of use. While the IARC collaborative study found a relative risk of 1.6 for long-term OC users with a significant trend, Moreno et al. observed a stronger association ( $\mathrm{OR}=4.0)$. In line with our findings, these two studies also found a reduced risk of cervical cancer for users who ceased OCs ( $\mathrm{RR}=0.8$ and $\mathrm{OR}=0.5$, respectively). Other prospective studies did not find associations between OCs and CIN3/CC risk [18-20]. Analyses combining duration and recency of use evaluated in our study found a trend of lower CIN3/CIS risk with cessation of use for both short and long-term OC users, reinforcing the null association among past users and the higher risk among current users. The IARC collaborative study has also analyzed these factors in combination, obtaining similar patterns of trend, but unlike our study, for both CIN3/CIS and ICC risk.

Since HPV is the necessary cause of CC, analyses including some measure of HPV infection are needed to assess potential residual confounding. In our nested case-control study we included the adjustment by HPV serology, and we evaluated OC use and CC risk among all cases and HPV seropositive controls. As already discussed, these results were broadly similar to those obtained in the cohort study, suggesting that we can reasonably rule out a large confounding effect due to HPV infection in our associations. In the last 10 years, most of the 
Table 3. Multivariate odds ratios for the association between factors related to endogenous and exogenous hormones and CIN3/CIS and ICC cases among all cases and HPV L1 seropositive control women in the nested case-control study.

\begin{tabular}{|c|c|c|c|c|}
\hline \multirow[t]{3}{*}{ Risk factor } & \multicolumn{4}{|c|}{ Among all cases and HPV L1 seropositive control women } \\
\hline & \multicolumn{2}{|c|}{ CIN3/CIS } & \multicolumn{2}{|c|}{ ICC } \\
\hline & Controls / Cases & OR $(95 \% \mathrm{Cl})$ & Controls / Cases & OR $(95 \% \mathrm{Cl})$ \\
\hline \multicolumn{5}{|l|}{ Number of FTP ${ }^{1}$} \\
\hline Never & $64 / 51$ & $1.0(\mathrm{ref})$ & $16 / 30$ & 1.0 (ref) \\
\hline Ever & $250 / 288$ & $2.0(1.2-3.2)$ & $122 / 131$ & $0.6(0.3-1.4)$ \\
\hline 1 & $71 / 71$ & $1.4(0.8-2.6)$ & $21 / 26$ & $0.9(0.3-2.6)$ \\
\hline 2 & $99 / 132$ & $2.3(1.3-4.0)$ & $69 / 53$ & $0.4(0.2-1.0)$ \\
\hline 3 & $47 / 46$ & $2.0(1.0-4.0)$ & $24 / 28$ & $0.7(0.3-2.0)$ \\
\hline$\geq 4$ & $24 / 28$ & $2.6(1.2-5.9)$ & $7 / 16$ & $1.2(0.3-4.6)$ \\
\hline$p$ for trend among ever ftp & & 0.2 & & 0.3 \\
\hline \multicolumn{5}{|l|}{ Number of induced abortions ${ }^{1,3}$} \\
\hline Never & $127 / 166$ & $1.0(\mathrm{ref})$ & $77 / 70$ & 1.0 (ref) \\
\hline Ever & $58 / 45$ & $0.5(0.3-0.8)$ & $29 / 41$ & $1.7(0.8-3.9)$ \\
\hline 1 & $42 / 36$ & $0.5(0.3-0.9)$ & $18 / 26$ & $2.0(0.8-5.0)$ \\
\hline$\geq 2$ & $16 / 9$ & $0.4(0.1-0.9)$ & $11 / 15$ & $1.4(0.5-4.3)$ \\
\hline \multicolumn{5}{|l|}{ IUD use us $^{2,3}$} \\
\hline Never & $143 / 160$ & 1.0 (ref) & $78 / 106$ & 1.0 (ref) \\
\hline Ever & 86 / 82 & $0.7(0.4-1.1)$ & $37 / 28$ & $0.6(0.3-1.2)$ \\
\hline \multicolumn{5}{|l|}{ OC use ${ }^{2}$} \\
\hline Never & $103 / 99$ & 1.0 (ref) & $59 / 56$ & 1.0 (ref) \\
\hline Ever & $214 / 244$ & $1.1(0.7-1.6)$ & $80 / 109$ & $1.7(0.9-3.3)$ \\
\hline Past & $182 / 197$ & $1.1(0.7-1.6)$ & $70 / 86$ & $1.6(0.8-3.1)$ \\
\hline Current & $26 / 41$ & $1.6(0.8-3.2)$ & $10 / 17$ & $2.7(0.8-9.1)$ \\
\hline \multicolumn{5}{|l|}{ Duration of OC use (years) ${ }^{2}$} \\
\hline Never & $103 / 99$ & 1.0 (ref) & $59 / 56$ & 1.0 (ref) \\
\hline$\leq 4$ & $82 / 82$ & $1.0(0.7-1.7)$ & $32 / 36$ & $1.4(0.6-3.0)$ \\
\hline $5-9$ & $53 / 54$ & $0.9(0.5-1.6)$ & $15 / 22$ & $2.1(0.8-5.5)$ \\
\hline$\geq 10$ & $60 / 94$ & $1.6(0.9-2.6)$ & $31 / 42$ & $1.8(0.8-4.2)$ \\
\hline$p$ for trend among OC users & & 0.1 & & 0.3 \\
\hline \multicolumn{5}{|l|}{ HT use ${ }^{4,5}$} \\
\hline Never & $84 / 83$ & 1.0 (ref) & $54 / 67$ & 1.0 (ref) \\
\hline Ever & $77 / 84$ & $1.0(0.6-1.7)$ & $39 / 18$ & $0.3(0.1-0.6)$ \\
\hline Past & $23 / 17$ & $0.6(0.3-1.4)$ & $10 / 8$ & $0.5(0.1-1.7)$ \\
\hline Current & $51 / 61$ & $1.2(0.7-2.2)$ & $29 / 10$ & $0.2(0.1-0.5)$ \\
\hline \multicolumn{5}{|l|}{ Duration of HT use (years) ${ }^{4,5}$} \\
\hline Never & $84 / 83$ & 1.0 (ref) & $54 / 67$ & 1.0 (ref) \\
\hline$\leq 1$ & $18 / 28$ & $1.4(0.6-3.0)$ & $21 / 11$ & $0.3(0.1-0.8)$ \\
\hline $2-4$ & $24 / 15$ & $0.5(0.2-1.2)$ & & \\
\hline$\geq 5$ & $28 / 19$ & $0.7(0.3-1.6)$ & $14 / 5$ & $0.2(0.05-0.8)$ \\
\hline$p$ for trend among menopausal hormones users & & 0.3 & & - \\
\hline
\end{tabular}

CIN3: cervical intraepithelial neoplasia grade 3; CIS: carcinoma in situ; ICC: invasive cervical cancer; OR: odds ratio; Cl: confidence interval; FTP: fullterm pregnancy; IUD: intrauterine device; OC: oral contraceptive; HT: hormone therapy. The number of cases and controls does not add up the total number because of missing values. Bold font indicates a statistically significant effect $(p<0.05)$.

${ }^{1}$ Unconditional regression models were adjusted by age, country, Chlamydia Trachomatis serology, Human herpesvirus 2 serology, body mass index, marital status, level education, physical activity, smoking habits, OC use and duration and menopausal status with HT use.

${ }^{2}$ Unconditional regression models were adjusted by age, country, Chlamydia Trachomatis serology, Human herpesvirus 2 serology, body mass index, marital status, level education, physical activity, smoking habits, number of full-term pregnancies and menopausal status with HT use.

${ }^{3}$ Excludes Bilthoven, Sweden and Norway because information was not collected for this variable.

${ }^{4}$ Unconditional regression models were adjusted by age, country, Chlamydia Trachomatis serology, Human herpesvirus 2 serology, body mass index, marital status, level education, physical activity, smoking habits, OC use and duration and number of full-term pregnancies.

${ }^{5}$ Among post and perimenopausal women (excluding premenopausal women).

doi:10.1371/journal.pone.0147029.t003 
published studies have also considered HPV infection in their analyses, and the results were globally comparable to those for all women. On the other hand, the very few studies that have used serology as HPV measurement found contradictory results [21-23]. Even though our study did not explicitly collect data related to sexual behavior we were able to adjust for serological markers of HPV, CT and HHV-2, as well as for marital status, which can all be considered good surrogate indicators of sexual behavior and risk of HPV exposure [24]. Since the results were very consistent with these adjustments, we were reassured that the potential effects of confounding by sexual behavior on our reported associations were minimal. Behavioral factors related to cervical cancer screening may also confound these associations. Again, even though the study did not collect individual data on screening practices, we systematically adjusted for surrogate markers of screening-related behavior such as number of pregnancies and variables related to contraceptive methods used in the past that may somewhat reduce the potential confounding effects due to screening practices. Also it is important to note that other studies that have adjusted for cervical screening did find positive associations with CC risk $[7,17]$.

A possible mechanism to explain the associations between OC use and CC risk is that estrogens and progestogens may interact with hormone receptors, mainly progesterone, present in cervical tissue and influence the natural history of HPV infection. Specifically, sex steroid hormones are thought to enhance the expression of HPV 16 E6 and E7 oncogenes stimulating the degradation of p53 tumor suppressor genes and enhancing the ability of the viral DNA to transform cells and induce carcinogenesis $[7,9,25,26]$. These potential mechanisms are somewhat consistent with data from transgenic mouse models showing that estrogen and its nuclear receptor promote CC in combination with HPV oncogenes, but there are not in line with the observation that progesterone inhibits cervical carcinogenesis in mice [27,28].

\section{Parity}

We found contrasting results between parity and risk of CIN3/CIS and ICC. Thus, while women with high parity had a higher risk of CIN3/CIS than nulliparous women, no associations were found with ICC. We also found that this association with high parity was present in each level of OC duration with a synergistic effect between the two variables. These results are in concordance with results from some $[20,23]$ but not all $[8,18,19,29]$ studies. The IARC collaborative study [8] and the IARC multicenter study [29] consistently found a significantly higher risk of CIN3/CIS and ICC among women with high parity. In contrast, two prospective studies conducted by Castle et al. [18,19] and a case-control study done in the US [23] did not find a significant association between parity and risk of CIN3/ICC. The lack of association between high parity and ICC risk found in our study could be explained by screening practices related to parity since it is likely that nulliparous women tend to be less screened than parous women. Hence, screening may act as a negative confounder, reducing the association between FTP and ICC risk. Globally the literature supports for an association between high parity and cervical cancer and pre-cancer risk.

As discussed in relation to OC use, these associations may be confounded by a number of other factors. However, adjustment by proxy measures of HPV exposure and sexual behavior did not change our risk estimates. In addition, two pooled analyses that adjusted their models by cervical screening practices obtained also similar results [8,29].

A possible biological mechanism for these associations could be that the elevated levels of estrogen and especially progesterone during pregnancy are responsible for the alterations in the squamo-columnal junction occurring during pregnancy, maintaining the transformation zone on the exocervix for many years. This would facilitate the direct exposure to HPV 
contributing to HPV persistence and progression to cervical neoplasia and cancer [8,20,29]. Another possible mechanism is immunosuppression linked to pregnancy which might enhance the role of HPV in cervical carcinogenesis [8].

\section{HT use}

Our findings provide evidence for a reduction in ICC risk among peri- and postmenopausal women using HT, an effect that was stronger with longer duration of use. We found very few studies evaluating HT and CC [26,30]. Overall, the literature rules out positive associations. If anything, most studies found weak inverse associations that rarely reached statistical significance. In assessing HT, however, it is important to consider the hormonal composition of these treatments. The IARC review reported an increased risk for breast and endometrial cancer in the 1970s among postmenopausal women using estrogen only therapy, and this triggered health authorities to modify the hormonal composition of HT [6]. Even though our data on HT formulation were very limited, menopausal estrogens alone were associated with an increased risk of CIN3/CIS and combined HT were inversely associated with ICC. Other studies found some evidence for a reduced risk of ICC with ever use of estrogen therapy as well as duration as compared with never use [30-32]. When HT also included progesterone, the inverse association with ICC risk still remained both in our study as well as in other studies $[30,33]$.

As noted previously, one of the limitations of this study is the lack of accurate individual screening history that may indeed influence the effects of HT use on CC risk. Thus, the inverse associations found with ICC, an outcome more susceptible to screening bias, could be somewhat overestimated. One possible explanation of this effect is that women who take HT are more frequently screened than non users, being consequently diagnosed and treated earlier of pre-cancerous lesions. Nevertheless, when we stratified analyses by proxy measures of screening such as parity and OC use, the inverse association remained (data not shown). The intrinsic mechanisms that might explain the biology of these potential associations are currently unknown. Data from HPV transgenic mouse models suggest that estrogens promote cervical carcinogenesis and progesterone inhibits CC [27,28]. However, our results, limited by the low number of subjects, are only partially consistent with these findings.

\section{Induced abortions}

An increased risk of ICC and a reduced risk of CIN3/CIS were found among women reporting an induced abortion. Globally, the literature with regard to cervical cancer and pre-cancer risk and induced abortions is inconclusive and our results were in line with some [24,34-36] but not all $[29,37,38]$ studies. More data are needed to better explore these potential associations.

\section{Menstrual lifespan}

We observed a decreased risk of both CIN3/CIS and ICC risk with increasing cumulative years of menstrual cycles. Longer menstrual lifespan is implicitly associated with shorter OC use duration and lower parity. However, the advantage of using menstrual lifespan is that includes in a single indicator combined information on the cumulative exposure to some endogenous and exogenous hormones taking into account the actual hormonal lifespan of the woman, providing thus more robustness in the risk estimates. It is interesting to note that even though this variable has been used in studies of other hormone-related cancers such as breast, ovarian and endometrial, no previous studies of CC have been identified in the literature. 


\section{IUD use}

We found a non significant decreased risk of both CIN3/CIS and ICC among IUD users in the nested case-control study that reached statistical significance in the analyses restricted to all cases and HPV seropositive controls when combining CIN3/CIS and ICC cases (OR $=0.7,95 \% \mathrm{CI}$ : 0.5-0.96; data not shown) and in the analyses restricted to HPV seropositive women for ICC risk (OR $=0.3,95 \%$ CI: 0.1-0.9; data not shown). The full case-control study and the different stratified analyses consistently found that IUD use was inversely associated with both CIN3/CIS and ICC risk. Few studies have evaluated associations between IUD use and CC risk with inconsistent results $[20,23,37,39]$. In a population-based study conducted in the US, Shields and colleagues found a protective effect between duration of IUD and ICC (OR $=0.3,95 \%$ CI: $0.2-0.8$, for $\geq 5$ years of IUD use) [23]. In a large pooled analysis, Castellsagué and colleagues reported a strong inverse association between IUD use and ICC ( $\mathrm{OR}=0.5,95 \%$ CI: 0.4-0.7) [39]. Other studies, however, have not confirmed these findings, although the use of a combined outcome (CIN3/CIS and ICC) or a low number of cases could explain these discrepancies [20,37]. Even though we adjusted for major risk factors such as past exposure to HPV, STIs and sexual behavior, it is true that we cannot completely rule out a residual confounding effect exerted by CC screening. It is important to note that the study by Castellsague did adjust the analyses for cervical HPV DNA, age at first sex, and number of PAP smears, and still found a statistically significant inverse association. One possible mechanism that could explain this potential protective effect is through a device-related inflammatory reaction in the cervix and endocervix that could influence the subsequent likelihood of HPV persistence and/or progression to CC [39].

\section{SCC versus adenocarcinoma}

Globally, the associations found between both exogenous and endogenous hormones and SCC risk were comparable to those obtained in models evaluating CIN3/CIS and ICC risk (data not shown). Concerning the risk of invasive adenocarcinoma, even though there were very few cases, an association was found with increasing number of FTP, in addition to OC use and HT, in the cohort study (data not shown). In concordance with our study, the IARC collaborative reanalysis evaluating risk factors for SCC and adenocarcinoma obtained an increased risk for each histological type with increasing duration of OC use [40]. Contrary to this and to another study [41], our results did not indicate that high parity is a risk cofactor for developing SCC, probably due to screening bias. Concerning HT use and contrary to our findings, Lacey and colleagues found that exogenous estrogens, especially unopposed estrogens, were positively associated with adenocarcinomas [30]. However, since both studies accounted for small number of cases, these findings should be cautiously interpreted.

\section{Strengths and limitations}

Our study has several strengths including its prospective, population-based design in most of the countries, a large sample size that embraces various regions in Europe, and the inclusion of two disease outcomes: CIN3/CIS and ICC. In addition, our inclusion of a nested case-control study within the cohort allowed us to determine and take into account biomarkers of past exposure to STIs such as HPV, CT and HHV-2.

Nevertheless, our study design failed to consider direct markers of HPV infection, cervical cancer screening practices or sexual behavior, not only at recruitment but also during followup, as none of the assessed risk factors or potential confounding variables was reassessed after recruitment. Since these risk factors could and most likely did change in an unknown direction during follow-up, our associations may have led to under- or overestimation of the real effects on CC risk. Another limitation was the low sensitivity of the HPV serology technique, as only 
half of infected women seroconvert, and a potential misclassification cannot be totally ruled out [12].

\section{Conclusions}

This study contributes strong evidence to consider high parity and long-term OC use important risk factors for cervical cancer and pre-cancer. These risk factors would act not independently but rather as cofactors that interact with HPV to induce cervical carcinogenesis. Our study also provides some evidence that IUD use and HT confer a reduced risk of cervical cancer, but these findings need further confirmation. Despite the clear involvement of hormones in cervical carcinogenesis, and from a public health point of view, adherence to current screening recommendations will certainly minimize the potential increased risk of CC associated with some of these hormonal risk factors.

\section{Supporting Information}

S1 Table. Baseline characteristics of cases and non-cases in the cohort study. (DOCX)

S2 Table. Risk of CIN3/CIS and ICC of the cervix according to the combined effect of number of full-term pregnancies and duration of use of hormonal contraceptives. (DOCX)

\section{S1 Text. STROBE checklist.} (DOC)

\section{Acknowledgments}

The authors would like to thank all EPIC participants and staff for their contribution to the study. The authors would like to thank Jennifer Vázquez for the laboratory analyses on CT and HHV-2.

\section{Author Contributions}

Conceived and designed the experiments: E. Roura NT TW SdS FXB MP VP EW NM JD ITG A. Tjønneland CM DP KTK KO FCC SM AF RTF JO AS A. Trichopoulou PL PO GM RT CS SP AM EL PHP HBBdM JRQ MJS CN AB NL JE DL AI RCT MAM MJG SR MT SF E. Riboli XC. Performed the experiments: E. Roura NT TW SdS FXB MP VP EW NM JD ITG A. Tjønneland CM DP KTK KO FCC SM AF RTF JO AS A. Trichopoulou PL PO GM RT CS SP AM EL PHP HBBdM JRQ MJS CN AB NL JE DL AI RCT MAM MJG SR MT SF E. Riboli XC. Analyzed the data: E. Roura NT XC. Contributed reagents/materials/analysis tools: E. Roura NT TW SdS FXB MP VP EW NM JD ITG A. Tjønneland CM DP KTK KO FCC SM AF RTF JO AS A. Trichopoulou PL PO GM RT CS SP AM EL PHP HBBdM JRQ MJS CN AB NL JE DL AI RCT MAM MJG SR MT SF E. Riboli XC. Wrote the paper: E. Roura NT TW SdS FXB MP VP EW NM JD ITG A. Tjønneland CM DP KTK KO FCC SM AF RTF JO AS A. Trichopoulou PL PO GM RT CS SP AM EL PHP HBBdM JRQ MJS CN AB NL JE DL AI RCT MAM MJG SR MT SF E. Riboli XC. Performed the lab assays: TW MP NM.

\section{References}

1. Ferlay J, Soerjomataram I, Dikshit R, Eser S, Mathers C, Rebelo M, et al. Cancer incidence and mortality worldwide: sources, methods and major patterns in GLOBOCAN 2012. Int J Cancer 2015; 136: E359-E386. doi: 10.1002/ijc.29210 PMID: 25220842 
2. Baseman JG and Koutsky LA. The epidemiology of human papillomavirus infections. J Clin Virol 2005; 32 Suppl 1:S16-S24. PMID: 15753008

3. Ho GY, Bierman R, Beardsley L, Chang CJ, Burk RD. Natural history of cervicovaginal papillomavirus infection in young women. N Engl J Med 1998; 338:423-8. PMID: 9459645

4. Cogliano V, Baan R, Straif K, Grosse Y, Secretan B, El Ghissassi F, WHO International Agency for Research on Cancer. Carcinogenicity of human papillomaviruses. Lancet Oncol 2005; 6:204. PMID: 15830458

5. Munoz N, Castellsague X, de Gonzalez AB, Gissmann L. Chapter 1: HPV in the etiology of human cancer. Vaccine 2006; 24 Suppl 3:S3-1-S310.

6. Combined estrogen-progestogen contraceptives and combined estrogen-progestogen menopausal therapy. IARC Monogr Eval Carcinog Risks Hum 2007; 91:1-528. PMID: 18756632

7. Appleby $P$, Beral V, Berrington dG, Colin D, Franceschi S, Goodhill A, et al. Cervical cancer and hormonal contraceptives: collaborative reanalysis of individual data for 16,573 women with cervical cancer and 35,509 women without cervical cancer from 24 epidemiological studies. Lancet 2007; 370:160921. PMID: 17993361

8. Cervical carcinoma and reproductive factors: collaborative reanalysis of individual data on 16,563 women with cervical carcinoma and 33,542 women without cervical carcinoma from 25 epidemiological studies. Int J Cancer 2006; 119:1108-24. PMID: 16570271

9. Rinaldi S, Plummer M, Biessy C, Castellsague X, Overvad K, Kruger KS, et al. Endogenous sex steroids and risk of cervical carcinoma: results from the EPIC study. Cancer Epidemiol Biomarkers Prev 2011; 20:2532-40. doi: 10.1158/1055-9965.EPI-11-0753 PMID: 21994406

10. Riboli E, Hunt KJ, Slimani N, Ferrari P, Norat T, Fahey M, et al. European Prospective Investigation into Cancer and Nutrition (EPIC): study populations and data collection. Public Health Nutr 2002; 5:111324. PMID: 12639222

11. Roura E, Castellsague X, Pawlita M, Travier N, Waterboer T, Margall N, et al. Smoking as a major risk factor for cervical cancer and pre-cancer: results from the EPIC cohort. Int J Cancer 2014; 135:453-66. doi: 10.1002/ijc.28666 PMID: 24338632

12. Castellsague X, Pawlita M, Roura E, Margall N, Waterboer T, Bosch FX, et al. Prospective seroepidemiologic study on the role of Human Papillomavirus and other infections in cervical carcinogenesis: evidence from the EPIC cohort. Int J Cancer 2014; 135:440-52. doi: 10.1002/ijc.28665 PMID: 24338606

13. Waterboer T, Sehr P, Michael KM, Franceschi S, Nieland JD, Joos TO, et al. Multiplex human papillomavirus serology based on in situ-purified glutathione s-transferase fusion proteins. Clin Chem 2005; 51:1845-53. PMID: 16099939

14. Sehr P, Muller M, Hopfl R, Widschwendter A, Pawlita M. HPV antibody detection by ELISA with capsid protein L1 fused to glutathione S-transferase. J Virol Methods 2002; 106:61-70. PMID: 12367730

15. Clifford GM, Shin HR, Oh JK, Waterboer T, Ju YH, Vaccarella S, et al. Serologic response to oncogenic human papillomavirus types in male and female university students in Busan, South Korea. Cancer Epidemiol Biomarkers Prev 2007; 16:1874-9. PMID: 17855708

16. Peters T, Brage S, Westgate K, Franks PW, Gradmark A, Tormo Diaz MJ, et al. Validity of a short questionnaire to assess physical activity in 10 European countries. Eur J Epidemiol 2012; 27:15-25. doi: 10. 1007/s10654-011-9625-y PMID: 22089423

17. Moreno V, Bosch FX, Munoz N, Meijer CJ, Shah KV, Walboomers JM, et al. Effect of oral contraceptives on risk of cervical cancer in women with human papillomavirus infection: the IARC multicentric case-control study. Lancet 2002; 359:1085-92. PMID: 11943255

18. Castle PE, Wacholder S, Lorincz AT, Scott DR, Sherman ME, Glass AG, et al. A prospective study of high-grade cervical neoplasia risk among human papillomavirus-infected women. J Natl Cancer Inst 2002; 94:1406-14. PMID: 12237286

19. Castle PE, Walker JL, Schiffman M, Wheeler CM. Hormonal contraceptive use, pregnancy and parity, and the risk of cervical intraepithelial neoplasia 3 among oncogenic HPV DNA-positive women with equivocal or mildly abnormal cytology. Int J Cancer 2005; 117:1007-12. PMID: 15986443

20. Jensen KE, Schmiedel S, Norrild B, Frederiksen K, Iftner T, Kjaer SK. Parity as a cofactor for highgrade cervical disease among women with persistent human papillomavirus infection: a 13-year followup. Br J Cancer 2013; 108:234-9. doi: 10.1038/bjc.2012.513 PMID: 23169283

21. Daling JR, Madeleine MM, McKnight B, Carter JJ, Wipf GC, Ashley R, et al. The relationship of human papillomavirus-related cervical tumors to cigarette smoking, oral contraceptive use, and prior herpes simplex virus type 2 infection. Cancer Epidemiol Biomarkers Prev 1996; 5:541-8. PMID: 8827359

22. Berrington A, Jha P, Peto J, Green J, Hermon C. Oral contraceptives and cervical cancer. Lancet 2002; $360: 410$. 
23. Shields TS, Brinton LA, Burk RD, Wang SS, Weinstein SJ, Ziegler RG, et al. A case-control study of risk factors for invasive cervical cancer among U.S. women exposed to oncogenic types of human papillomavirus. Cancer Epidemiol Biomarkers Prev 2004; 13:1574-82. PMID: 15466972

24. Syrjanen K, Shabalova I, Petrovichev N, Kozachenko V, Zakharova T, Pajanidi J, et al. Oral contraceptives are not an independent risk factor for cervical intraepithelial neoplasia or high-risk human papillomavirus infections. Anticancer Res 2006; 26:4729-40. PMID: 17214333

25. Moodley M, Moodley J, Chetty R, Herrington CS. The role of steroid contraceptive hormones in the pathogenesis of invasive cervical cancer: a review. Int J Gynecol Cancer 2003; 13:103-10. PMID: 12657108

26. Gadducci A, Barsotti C, Cosio S, Domenici L, Riccardo GA. Smoking habit, immune suppression, oral contraceptive use, and hormone replacement therapy use and cervical carcinogenesis: a review of the literature. Gynecol Endocrinol 2011; 27:597-604. doi: 10.3109/09513590.2011.558953 PMID: 21438669

27. Chung SH, Franceschi S, Lambert PF. Estrogen and ERalpha: culprits in cervical cancer? Trends Endocrinol Metab 2010; 21:504-11. doi: 10.1016/j.tem.2010.03.005 PMID: 20456973

28. Yoo YA, Son J, Mehta FF, DeMayo FJ, Lydon JP, Chung SH. Progesterone signaling inhibits cervical carcinogenesis in mice. Am J Pathol 2013; 183:1679-87. doi: 10.1016/j.ajpath.2013.07.026 PMID: 24012679

29. Munoz N, Franceschi S, Bosetti C, Moreno V, Herrero R, Smith JS, et al. Role of parity and human papillomavirus in cervical cancer: the IARC multicentric case-control study. Lancet 2002; 359:1093-101. PMID: 11943256

30. Lacey JV Jr., Brinton LA, Barnes WA, Gravitt PE, Greenberg MD, Hadjimichael OC, et al. Use of hormone replacement therapy and adenocarcinomas and squamous cell carcinomas of the uterine cervix. Gynecol Oncol 2000; 77:149-54. PMID: 10739704

31. Adami HO, Persson I, Hoover R, Schairer C, Bergkvist L. Risk of cancer in women receiving hormone replacement therapy. Int J Cancer 1989; 44:833-9. PMID: 2583865

32. Parazzini F, La Vecchia C, Negri E, Franceschi S, Moroni S, Chatenoud L, et al. Case-control study of oestrogen replacement therapy and risk of cervical cancer. BMJ 1997; 315:85-8. PMID: 9240046

33. Schneider C, Jick SS, Meier CR. Risk of gynecological cancers in users of estradiol/dydrogesterone or other HRT preparations. Climacteric 2009; 12:514-24. doi: 10.3109/13697130903075352 PMID: 19905903

34. Parazzini F, La Vecchia C, Negri E, Cecchetti G, Fedele L. Reproductive factors and the risk of invasive and intraepithelial cervical neoplasia. Br J Cancer 1989; 59:805-9. PMID: 2736217

35. La Vecchia C, Negri E, Franceschi S, Parazzini F. Long-term impact of reproductive factors on cancer risk. Int J Cancer 1993; 53:215-9. PMID: 8425757

36. Deacon JM, Evans CD, Yule R, Desai M, Binns W, Taylor C, et al. Sexual behaviour and smoking as determinants of cervical HPV infection and of CIN3 among those infected: a case-control study nested within the Manchester cohort. Br J Cancer 2000; 83:1565-72. PMID: 11076670

37. Zondervan KT, Carpenter LM, Painter R, Vessey MP. Oral contraceptives and cervical cancer-further findings from the Oxford Family Planning Association contraceptive study. Br J Cancer 1996; 73:12917. PMID: 8630295

38. Russo E, Kupek E, Zanine RM. Vaginal delivery and low immunity are strongly associated with highgrade cervical intraepithelial neoplasia in a high-risk population. J Low Genit Tract Dis 2011; 15:195-9. doi: 10.1097/LGT.0b013e31820918ea PMID: 21436727

39. Castellsague X, Diaz M, Vaccarella S, de Sanjose S, Munoz N, Herrero R, et al. Intrauterine device use, cervical infection with human papillomavirus, and risk of cervical cancer: a pooled analysis of 26 epidemiological studies. Lancet Oncol 2011; 12:1023-31. doi: 10.1016/S1470-2045(11)70223-6 PMID: 21917519

40. Comparison of risk factors for invasive squamous cell carcinoma and adenocarcinoma of the cervix collaborative reanalysis of individual data on 8,097 women with squamous cell carcinoma and 1,374 women with adenocarcinoma from 12 epidemiological studies. Int J Cancer 2007; 120:885-91. PMID: 17131323

41. Castellsague X, Diaz M, de Sanjose S, Munoz N, Herrero R, Franceschi S, et al. Worldwide human papillomavirus etiology of cervical adenocarcinoma and its cofactors: implications for screening and prevention. J Natl Cancer Inst 2006; 98:303-15. PMID: 16507827 